\title{
EL CINE Y SU USO COMO HERRAMIENTA DE TRATAMIENTO EN TERAPIA OCUPACIONAL
}

\author{
THE USE OF FILMS AS A TREATMENT TOOL IN OCCUPATIONAL THERAPY
}

\section{Paula Milenka Cancino González( $\left.{ }^{1}\right)$}

\section{Resumen}

Este ensayo ha sido confeccionado para la obtención del título profesional de Terapeuta Ocupacional, en el marco del examen de pre- título exigido por la Universidad de Chile. Su objetivo es fundamentar el uso del cine como herramienta de intervención, otorgándole una utilidad explícita desde la Terapia Ocupacional (desde ahora T.O.), que se vea concretizada en una propuesta de aplicación. Su temática aborda la relación del cine con esta disciplina, su fundamento desde la Ciencia de la Ocupación, también propone la idea del uso de las películas en la intervención no sólo desde su proyección sino desde su creación, reflexiona acerca de las contribuciones pendientes de los Terapeutas Ocupacionales en su desarrollo y razona sobre la utilidad de las riquezas del recurso cinematográfico en la intervención profesional.

Palabras clave:

Cine, Terapia Ocupacional, películas, herramienta de intervención.

\begin{abstract}
This paper has been made in order to obtain the professional qualification of Occupational Therapist, an examination of prequalification required by Universidad de Chile. Its aim is to justify the use of movies as a tool for intervention, providing an explicit usefulness in Occupational Therapy (OT from now on), to be settled into a proposed application. The paper addresses the relationship between cinema and the discipline, its rationale from Occupational Science, and also proposes the idea of using movies in the intervention not only from its projection but since its inception; reflects on pending contributions from occupational therapists in their development, and reasons about the utility of the wealth of cinematographic resource in professional intervention.
\end{abstract}

Keywords:

Film, Occupational Therapy, Movies, Tool for intervention. Introducción: Cine y su uso.

\footnotetext{
${ }^{1}$ Terapeuta Ocupacional, Bachiller en Humanidades y Cs. Sociales. Contacto: paulamilenka@ gmail.com. Fono : 26014021 -
} (7)9578972 . 


\section{InTRODUCCIÓN: CINE Y SU USO}

El cine es mucho más que 24 fotogramas por segundo, es arte, expresión y creación, es un conjunto de procesos y elementos culturales, institucionales y actorales que construyen discursos, imágenes, historias, valores, ideas, representaciones, interpretaciones, identidades y símbolos. Es un medio de comunicación de grandes masas, según Jorge Letelier², en el año 2011 el público chileno asistente al cine fue 16.946.240 espectadores; ha de considerarse que este número no contempla otras formas de acceso a las películas, como el arriendo, su presentación en la televisión o su compra en DVD, además de las vías informales de distribución y acceso como las compra/venta de películas "piratas" y la descarga desde la web o su reproducción "online". Queda en evidencia que en nuestro país, ver películas se constituye como una actividad de tiempo libre realizada por la gran mayoría de la población.


Si bien el cine se reconoce como un medio de entretenimiento, también ha sido utilizado en disciplinas tales como psicología y educación; estas mayormente, han desarrollado el uso del cine como herramienta desde sus prácticas profesionales; ejemplo de esto es la re-significación de los hechos traumáticos acontecidos por víctimas de violencia intrafamiliar ${ }^{3}$, la víctima se ve identificada y proyectada tanto en el personaje como en la situación, universaliza y mira sus vivencias desde una nueva perspectiva, identificando, reflexionando y replanteándose soluciones (Armijo, 2010). Por otra parte en educación, "posee un gran potencial para la didáctica de lenguas y culturas" (Costa, 2005/2006); también ha servido como fuente de información de datos históricos, políticos y sociológicos, o de temáticas que el profesorado quiera abordar dependiendo de los objetivos que tengan y del público a quien se proyecta.

Si el cine ha sido útil en estas disciplinas, como herramienta de tratamiento o recurso didáctico, ¿qué hay de su uso en T.O.?, ¿será justificable su uso en esta disciplina?, y si lo es, ¿cómo se podrá aplicar?

\footnotetext{
Nota periodística publicada en el diario La Tercera, titulada: "Cine Chileno del 2011, el año de la recuperación, pero las dudas persisten".
}

Tesis para optar al grado de magíster en psicología clínica de adultos, escrita por Ps. Nathaly Armijo Richamont.
El hecho de que el cine sea fuente de diversión para la mayoría de los chilenos, ha de ser razón suficiente para que se vuelva foco de T.O., no sólo por formar parte de las ocupaciones de la población sino que también por el aporte que se puede hacer sobre su uso, ya que, como se indica en el "Marco de trabajo para la Práctica de la T.O.: Dominio y Proceso", proveer intervención de T.O. puede involucrar el uso terapéutico de la ocupación como un medio o como método para modificar el desempeño y su finalización ocurre cuando el cliente mejora su capacidad para involucrarse en actividades significativas (AOTA $\left.{ }^{4}, 2002\right)$.

"Actualmente, hay una escasez de investigación sobre la eficacia del uso del cine como técnica terapéutica" (Armijo, 2010). Es exigua la contribución que se ha hecho desde T.O. en este ámbito y siendo esta una profesión en desarrollo debería ser lo contrario. Si se busca información sobre cine y T.O. es poco lo que se encuentra sobre una aplicación fundamentada, las temáticas sólo alcanzan el análisis ocupacional del personaje de alguna película o la representación de las y los T.T.O.O. en las cintas, es escaso el material redactado por estos profesionales sobre el cine y su

\footnotetext{
4 Asociación Americana de Terapia Ocupacional, Revista Americana de Terapia Ocupacional, traducción y adaptación al español por $M^{a}$ Elena Riveros E.
} 
utilidad, y más escaso aún desde los T.T.O.O. latinoamericanos, lo cual incurre en el desaprovechamiento de una ocupación que serviría como herramienta para modificar el desempeño humano y sus efectos sobre la discapacidad y la enfermedad. Desde la experiencia de la autora de este ensayo, la proyección de películas tiene cabida en intervención grupal de usuarios, bajo el marco de actividad de tiempo libre en T.O. y con los simples propósitos de proveer un momento de distracción o de entretenimiento. Por ejemplo se vio utilizado en un programa de tiempo libre saludable con adultos mayores, en donde ellos elegían qué películas ver, evocando con emoción recuerdos que estas les traían. "Si principalmente es a través de la imagen que, al comunicar el mensaje, afecta al espectador y puede llegar a influirlo de tal manera que se produzca un cambio en él" (Armijo, 2010), es razonable pensar en cómo afectaría a una persona transmitir el mensaje por este medio y presentárselo a los demás, es decir, usar el cine como herramienta terapéutica no sólo desde su proyección sino que desde su creación.

\section{Desarrollo temático: Creación de cine como herramienta terapéutica}

\section{Recursos cinematográficos}

Las películas se tornan parte de la cultura, generando imágenes y símbolos que entran en el conocimiento de la mayoría de la población, por lo que generar proyectos de cine con identidad cultural propia, que abarquen la problemática que convoca a los usuarios a un tratamiento o un tema de importancia para ellos, implica un recurso terapéutico capaz de producir efectos a nivel personal y social. La creación de un filme conlleva adquirir diferentes conocimientos, realizar decisiones a nivel grupal, ejercitar la capacidad de expresar ideas y sentimientos, generar un mensaje con propósito y reflexionar acerca de la temática representada, principalmente se trata de comunicar. "Aunque es una aventura terapéutica más entusiasta, es extremadamente efectiva como proyecto metafórico visual, auditivo y quinésico (Arnott and Gushin, 1976)" (Fazio, 1992). Utilizar la metáfora en el tratamiento permite acceder a la condición humana de una manera gráfica, otorgando una representación simbólica a la subjetividad de las personas; lo que permitiría no sólo que los usuarios reflexionen y organicen sus pensamientos frente a la temática, sino que también creen la imagen que quieren que los demás vean. "La narrativa y la metáfora permiten a las personas la oportunidad de distanciarse de los acontecimientos, de sus propias experiencias y convertirse en el protagonista de sus propios relatos de vida" (Armijo, 2010).

Para realizar la producción cineasta-gráfica, dentro del marco de tratamiento de T.O., se debe tener un conocimiento sobre el modo de generar cine y las características de este, así se podrá utilizar cada elemento de la creación del filme asertivamente hacia los objetivos del tratamiento. "No podemos dejar al azar estrecho de mirar sin saber que miramos (Alba, 2007)" (Lorente, 2011); los usuarios, al saber lo que ven y cómo se utilizan los recursos cinematográficos, enriquecen su capacidad para contar una historia, pues las técnicas fílmicas serían ocupadas para generar y dar carácter al mensaje que se quiere entregar. Si el objetivo es contar una historia, que puede contener la problemática de los usuarios o una temática relevante para ellos, los recursos que otorga el cine deben ser utilizados para que esta comunique no sólo el relato de hechos y acciones de los personajes, sino que también los valores, las emociones y creencias asociadas a estos, es decir, en esta actividad deben quedar representados tantos los aspectos subjetivos como objetivos. Es necesario, entonces, adentrarse en el lenguaje cinematográfico y conocer elementos básicos como el plano, el montaje, los movimientos de la cámara, el tratamiento del tiempo y el espacio, la profundidad del campo, el eje, la luz, la música, las transiciones, el guión y el argumento. "Una obra de cine ficción es el resultado de un conjunto de tareas que se inician con un guión, una selección de actores, una búsqueda de locaciones y una adecuada ambientación" (Chion 1992; Gamerro y Salomon 1993). A esta etapa prefílmica le sigue el rodaje, momento en el que se produce el material audiovisual que finalmente será utilizado en la edición o montaje (Eisenstein 1977; Pudovkin 1999; Villain 1994)" (Gallardo, 2008). Para iniciar el relato es necesario saber qué y cómo se quiere comunicar; para esto, los usuarios deberán determinar la trama, el argumento, la vestidura, la investidura, la diégesis y la narración. Decididas estas características la creación cinematográfica se debe estructurar con su forma clásica: la exposición, el desarrollo (clímax) y el desenlace. Los elementos anteriores son de importancia para T.O. pues constituyen el lenguaje con el que se comunicará y manipulará el mensaje de los usuarios, saber ocuparlo será responsabilidad del profesional para guiar a los participantes y dar coherencia entre la intención comunicacional y el producto cinematográfico. 
Análisis del cine desde T.O.
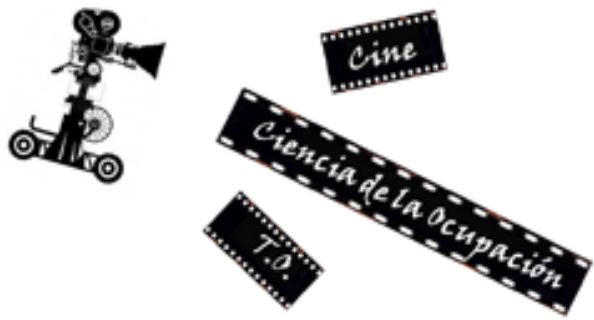

¿Cómo fundamentar el uso del cine en T.O.? Analizando los elementos que conforman una creación cinematográfica, tanto aquellos que se exponen en la cinta, como aquellos anexos para su realización; los que son parte de un entramado de acciones, roles, habilidades, contextos y demandas ocupacionales.

Existe un fundamento desde la Ciencia de la Ocupación que habla de la apropiación estética de la actividad ocupacional, correspondiendo a la contemplación final del trabajo realizado que nos otorga satisfacción; en la producción de cine, este elemento sería esencial, la contemplación de la película y su exposición a un público, son etapas Ilenas de emoción que permiten "cerrar" un proceso con un alto nivel de placer y regocijo. Dos filósofos se refieren a la estética en la ocupación, avalando con mayor profundidad la importancia de esta en la intervención a través de la cinematografía, primero, "Platón explica que desde las artes llega una brisa que trae salud a cada lugar preciso que el cuerpo físico o el alma humana necesita." (Gómez y Rueda, 2002). Esto hace referencia a los beneficios individuales en la salud; segundo, "Aristóteles expresó que el arte surge cuando muchas nociones obtenidas de la experiencia sensible producen un solo juicio universal sobre las cosas semejantes... nos hace conocer más nuestra realidad objetiva y subjetiva como una sola." (Góméz y Rueda, 2002). Ésta reflexión señala los beneficios sociales de coherencia o de acuerdo y entendimiento sobre las temáticas expuestas en los filmes.

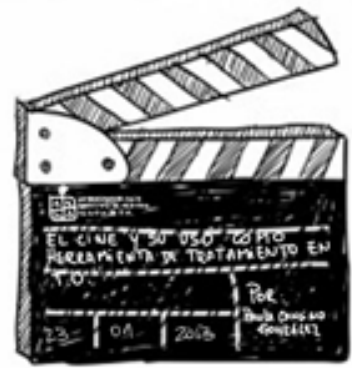

Crear cine es una ocupación, esto se justifica en su coherencia con la definición: "Actividades de la vida diaria, nombradas, organizadas y a las que se les otorga significado y valor por parte del individuo y la cultura. La ocupación es todo lo que las personas hacen para ocuparse a sí mismos, incluyendo actividades de autocuidado... disfrute de la vida... y contribuyendo a la construcción social y económica de sus comunidades (Law, Polatajko, Baptiste y Towsend, 1997)" (AOTA, 2002). Dependiendo de su enfoque, puede clasificarse dentro de las áreas de ocupación tales como: educación, trabajo, tiempo libre y participación social. Además, su producción está precedida, seguida y en paralelo con diferentes ocupaciones y propósitos, directa e indirectamente relacionados con la creación de la película. El uso de la producción fílmica es compatible con el dominio de la T.O., se fundamenta porque contiene las áreas de la experiencia humana en las que los profesionales de T.O. ofrecen asistencia, ya que en el contexto de intervención, tiene demandas de utilización de objetos como cámaras, luces, vestuarios, escenografías; de espacios como locaciones, iluminación y decoración de escenarios; posee demandas sociales como el trabajo en equipo y la contribución ciudadana de entregar un mensaje a través del arte, de secuencias y tiempos que no sólo tienen relación con la organización de la producción, sino con la manipulación temporal de la ficción; y demandas de estructuras y funciones corporales que se requieren al actuar, grabar, debatir, pensar, crear al comunicar, entre otras acciones.

La utilidad explícita de hacer filmes en T.O. radica en la consecución de objetivos terapéuticos que a través de estos se podrían lograr, así es como las habilidades de desempeño se ejercitarían continuamente durante el desarrollo del proyecto cinematográfico, con mayor o menor énfasis en las distintas etapas, pero siempre requerirá del uso de habilidades motoras, de procesamiento, comunicación e interacción ${ }^{5}$.

El cine es versátil, no es necesario que todos los participantes del grupo realicen todas las actividades que se contienen en su creación. Luego de una evaluación, los o las T.T.O.O. deben otorgarle roles a los usuarios dentro de la producción, considerando sus motivaciones e intereses, como también la problemática que se quiere trabajar a través del cine, por ejemplo, para un usuario que requiera entrenar la movilidad en la comuna, será

4 Clasificación de las habilidades del desempeño según el "Marco de Trabajo para la Práctica de la T.O.", AOTA (2002). 
de utilidad que sea parte del equipo que se traslade a las locaciones de grabación, como camarógrafo, actor o con otra responsabilidad pertinente; en este ejemplo se observa que a pesar de que el cine no se clasifica como una actividad de la vida diaria instrumental, en su producción sí se contiene; son muchos los roles (guionista, director, actor, entre otros) y las áreas de la ocupación (AVDB-I, educación, trabajo, juego, tiempo libre y participación social) que tienen cabida en la producción de cine, lo que se puede utilizar para la obtención de objetivos terapéuticos, estos a su vez pueden ser variados y adaptados a las necesidades de la población de usuarios. Sin embargo hay beneficios comunes a todos los participantes independiente de la temática del proyecto, como es la creatividad; entendida no como un "simple privilegio de la imaginación fantaseadora ni de la sensibilidad afectiva; la inteligencia, la percepción, el esfuerzo por objetivar, la capacidad de comunicación, de innovación, la resolución de problemas, el pensamiento divergente, la apertura al cambio, la interrogación, la observación, la experimentación, etc., son procesos creativos" (Granados, 2009). Junto a la creatividad, está el ejercicio de la narración, que desarrolla habilidades de observación y organización, aplicables a la resolución de conflictos en diferentes ámbitos de la vida cotidiana, tanto a nivel subjetivo como en acciones observables, el trabajo en equipo o el análisis del contexto cercano son ejemplos de estas.

El material audiovisual creado por los usuarios, responde a una sensibilidad personal frente a lo que perciben y a conocimientos técnicos propios del montaje visual, primando una adecuada planificación y trabajo colaborativo (Bustos, 2010). Por otra parte, está el desarrollo de la ciudadanía, pues a través de la expresión artística se cruzan problemáticas sociales, políticas, económicas, morales, éticas, de salud y de emociones que involucran a la sociedad entera, transmitiendo un mensaje de concientización y conocimiento desde la perspectiva de los usuarios; se promueven "valores humanizadores, de manera transversal e interdisciplinar y competencial, para contribuir a desarrollar valores y elementos de identidad personal y de pertenencia" (Ambròs y Breu, 2008). Otro beneficio de la creación cinematográfica radica en los recursos que le otorga a los y las T.T.O.O., entre ellos, la manera de obtener información acerca de los usuarios, sobre sus sistemas familiares, relaciones interpersonales, creencias, valores, ideologías, expectativas personales, el concepto de sí mismo y de sus problemáticas, entre otros datos, que dependerán del uso profesional que le den a la herra- mienta, además mediante la creación del filme se genera un contexto sutil para tratar las temáticas que causan el problema en el desempeño ocupacional de cada usuario, variando la expectación por la creación, "esta terapia permite introducir las temáticas de una forma más sutil, donde el espectador no se siente amenazado, pues está viendo en primera instancia una historia ajena, pero que a la larga identificará con sus vivencias" (Armijo, 2010). "El cine es un arte, por lo cual todo lo que está en el mundo le pertenece como material de trabajo, placer y reflexión (Debrix, 1997)" (Gallardo, 2008); es así como las dificultades del desempeño ocupacional o bien las preocupaciones e intereses que los usuarios requieran comunicar son una temática pertinente a desarrollar en un proyecto fílmico; aquí se observa la utilidad explícita de este recurso en T.O. al ser una alternativa artística para desarrollar la dinámica entre el usuario, el contexto y la actividad. Como indica Armijo $^{6}$ (2010), "el arte es una vía para el reencuentro con uno mismo y, por lo tanto, con un sentido sanador, como bien lo plantean muchos especialistas, entre ellos, Jodorowsky (Ortega, 2008); en la frase 'un arte que no sana no es arte'"

Comprueba la utilidad de esta herramienta la experiencia de la T.O. Helen Mason, fundadora y líder del equipo de Animation Therapy $\mathrm{Ltd}^{7}$. Ella junto a su equipo de trabajo, utilizan la creación de películas de animación (stop motion) como herramienta de T.O., envolviendo procesos complejos que pueden ser clasificados para ayudar a los usuarios a lograr niveles de desempeño ocupacional óptimo. Entre los beneficios obtenidos señala que atraviesa brechas generacionales, entrena habilidades de desempeño de forma individual o grupal, habilita el flujo y el compromiso con el tratamiento y explora las relaciones interpersonales. Como proceso grupal con diferentes roles que contribuyen al filme o al producto, el terapeuta puede adaptar las técnicas para fines individuales según los intereses únicos de la persona; dentro del marco institucional, por ejemplo, la animación permite a las personas proyectarse en un mundo de imaginación que les ofrece esperanza y los prepara para el cambio, también facilita tiempo

\footnotetext{
6 Psicóloga. Autora de la tesis para optar al grado de magíster en psicología clínica de adultos, "Uso de la Cineterapia en Mujeres Víctimas de Violencia Intrafamiliar".

Para más información visitar http://www.animationtherapy.co.uk/ public-home.aspx
} 
y seguridad para explorar la relación terapéutica y la expresión de sí mismo dentro de ella.

\section{Aplicación del cine en T.O.}

El proceso de intervención en T.O. se divide en tres sub-etapas correspondiendo al plan, implementación y revisión de la intervención (AOTA, 2002); para que la creación de cine tenga el carácter de tratamiento de T.O. debe adaptarse a estas etapas, a la realidad del contexto en el que se lleve a cabo y a las necesidades y características (principalmente rango etario y problemática ocupacional) de la población con la que se trabajará en conjunto.

El plan de intervención debe considerar todas las etapas del proyecto cinematográfico, partiendo por la preparación y motivación de los usuarios, donde estos adquirirán los conocimientos básicos de la técnica cinematográfica y se relacionarán críticamente con la proyección de películas; posteriormente debe continuar una etapa de debate grupal, en donde se decidirá la temática que se quiere representar en el filme, seguida de una etapa creativa en donde se deberá generar un guión. Luego se puede iniciar una serie de acciones que dependerán de los recursos con los que se cuente y de la intención comunicacional de los usuarios; los y las T.T.O.O. deben conocer las posibilidades reales de la producción en tanto recursos y habilidades con las que se cuenta para que el proyecto tenga reales posibilidades de llevarse a cabo. Dentro de las acciones que siguen está el decidir qué tipo de filme será y el carácter de la narrativa del guión, luego se dará paso a la confección, recaudación, acceso y elección de recursos como el vestuario, las locaciones o escenarios, los actores o personajes principales y el manejo de herramientas como cámaras, iluminación y audio. Sobre el costo de la actividad, dependerá de los recursos con los que se cuente y el proyecto que se quiere lograr, lo que puede ir desde costo cero, utilizando cámaras simples como la de un celular o de uso personal, hasta necesitar recursos de mayor precio, los que se pueden conseguir de variadas formas, recaudando dinero o participando en fondos concursables. Posteriormente, viene la etapa del rodaje, seguida de la edición y el montaje en donde se necesitarán conocimientos computacionales. Finalmente, se debe planificar cuál será la audiencia de la película producida; esto debe decidirse junto a los usuarios, y en conocimiento de las posibilidades que se ofrecen en las redes y organizaciones sociales, estos espacios de proyección pueden abarcar desde mostrar la película a una población cercana como familiares y amigos, organizar una proyección a nivel vecinal/comunal, hasta participar en concursos (como los ofrecidos por el INJUV ${ }^{8}$ y/o por el FENSACOR $^{9}$ ) o distribuirla en un medio gratuito y masivo como Youtube ${ }^{10}$; esto requiere que el o la T.O. tengan conocimiento de las redes que se pueden ocupar para la exposición del proyecto. El desarrollo del plan está precedido por una etapa de evaluación, que permite establecer objetivos; estos deben coincidir con los intereses de cada usuario y del grupo, para esto el rol y responsabilidades que tenga cada persona en la producción serán de fundamental importancia. Ejecutar la intervención requerirá de la flexibilidad y creatividad de los usuarios para modificar acciones planeadas en un principio, según los recursos y acceso a herramientas y localidades reales. La revisión de la intervención podrá hacerse a nivel grupal e individual, no se debe olvidar que el objetivo fundamental es que las personas se mantengan activas a través de la ocupación.

\section{Conclusiones}

La siguiente reflexión nos lleva a visualizar a T.O. como una profesión con una contribución pendiente en el desarrollo del cine como una herramienta terapéutica; en este escrito se justifica su uso desde la utilidad explícita de los elementos y procesos cinematográficos que corresponden a un entramado de acciones, roles, habilidades, contextos y demandas ocupacionales; a través de ellos se cumplirían objetivos terapéuticos, que pueden ser variados y adaptados a las necesidades de la población de usuarios a nivel grupal e individual, esperando beneficios en el desempeño ocupacional de estos; dentro de sus beneficios, posibilita el entrenamiento de habilidades motoras, de procesamiento y de comunicación e interacción, permite el desarrollo de la creatividad, de la narración y de la participación ciudadana, por lo que

\footnotetext{
8 Instituto Nacional de la Juventud (INJUV) es un organismo de servicio público encargado de colaborar con el Poder Ejecutivo en el diseño, planificación y coordinación de las políticas relativas a los asuntos juveniles.

9 Festival Chileno Internacional del Cortometraje de Santiago.

10 YouTube (pronunciación AFI) es un sitio electrónico en el cual los usuarios pueden subir y compartir videos. Visitar www.youtube.com
} 
se fundamenta su uso en T.O. y se recalca la necesidad de que los profesionales de esta disciplina contribuyan a su desarrollo. Al teorizar acerca de una aplicación del cine con perspectiva ocupacional, se observa el requerimiento de mayor profundización e investigación para conocer la verdadera riqueza del cine como instrumento terapéutico. Además, es necesario que los profesionales adquieran conocimientos sobre las técnicas del cine para proveer de un tratamiento serio, usando sus recursos de manera provechosa. Con estas necesidades expuestas, dentro de las proyecciones que tendría el desarrollo de la creación cinematográfica en la práctica de Terapia Ocupacional, están las de generar instancias de investigación sobre la real implicancia del cine en la intervención con diferentes tipos de poblaciones de usuarios, que varíen tanto en edades, como problemáticas, ya sean del área social, mental, física e intelectual. Al sistematizar dichas intervenciones y generar nuevos conocimientos y experiencias, que nos indiquen claramente cómo manejar cada intervención según el caso y nos demuestren qué tipo de poblaciones son más capaces de desarrollar un proyecto con estas demandas y qué adaptaciones son recomendadas; el siguiente paso sería crear cursos en donde los T.T.O.O. puedan aprender dichas prácticas, asociando los recursos cinematográficos con las dificultades específicas de cada población con el propósito de que el objetivo terapéutico se mantenga claro, definido y se alcance sin perder el arte del proceso, al mismo tiempo es importante que la creatividad no se vea alterada negativamente por la falta de conocimientos.

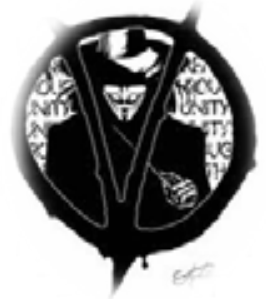

Por otra parte una de las grandes contribuciones fuera de la consecución del objetivo terapeútico y el progreso individual de los participantes, es la generación de identidad cultural propia, es decir, al crear imágenes y símbolos con significados personales, grupales, comunales y hasta nacionales, nos estaríamos haciendo cargo de un mensaje potente que puede influenciar, generar o cambiar paradigmas establacidos, en pro de la población comunicadora.

Acercar el arte a las personas, es más que conocer, es hacer.

\section{FUENTES DE INVESTIGACIÓN DOCUMENTAL}

Ambròs, A. y Breu, R. (2011). Cine y ciudadanía: encrucijada de

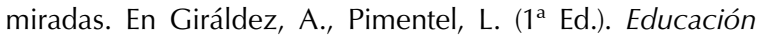
artística, cultura y ciudadanía. De la teoría a la práctica. (pp. 115-126) Madrid: Organización de Estados Iberoamericanos para la Educación, la Ciencia y la Cultura (OEI).

Armijo, N. (2010). Uso de la Cineterapia en Mujeres Víctimas de Violencia Intrafamiliar. Tesis para optar al grado de magíster en Psicología de adultos. Universidad de Chile.

Betanzo, P. B. (2010). Educación Artística. El Cine como Herramienta eficaz para un aprendizaje concreto, activo y reflexivo: una experiencia en Aula. Congreso Iberoamericano de Educación (págs. 1-12). Buenos Aires.

Conejo, I. M. ( 2009, diciembre). Interrelaciones entre la Creatividad, el Arte, la Educación y la Terapia. Arte y Movimiento, $N^{\circ} 1,51-62$.

Cortínez, V. ¿Es posible hacer cine en Chile? Las hazañas del Gringuito de Sergio Castilla. Perspectivas, 271-284.

Costa, E. (2005/2006). La pantalla en las aulas: el cine como fin específico en la adquisición de segundas lenguas. Revista de Lenguas para Fines Específicos, 95-112.

Fazio, L. S. (1992). Tell Me a Story: The Therapeutic Metaphor in the Practice of Pediatric Occupational Therapy. The American Journal of Occupational Therapy, Volume 46, N²2, 112-119.

Gallardo, F. (2008). Elementos para una Antropología del Cine: Los Nativos en el Cine Ficción de Chile. Revista de Antropología Chilena, volumen 40, número especial, 317-325.

Gómez, S. y Rueda, L. (2002). Bases para el desarrollo de la ciencia de la Ocupación.

Lauretti,P.; González, L. y Flores, Y. (2009, mayo/agosto). Cine interactivo como estrategia de intervención grupal. En/@ce: Revista Venezolana de información, Tecnología y Conocimiento, 57-69.

Lorente, P. (2011). Literatura universal y cine. Apuntes para un uso razonado del cine en las aulas. Universidad de Zaragoza, Facultad de Educación.

Mason, H. R. (2009, November). Dare to Dream: The use of animation in Occupational Therapy. Mental Health Ocupational therapy, Volume 14 Issue 3, 111-115.

Pardo, A. (2011). Autoaprendizaje experiencial y cine: el caso de la película "Paraíso Travel". Arte, Individuo y Sociedad, 55-67.

Pierce, D. (2005, January/February). The Usefulness of Video Methods for Occupational Therapy and Occupational Science Reserch. The American Journal of Occupational Therapy, volumen 59, № 1, 9-19.

Stange, H.; Salinas, C. (2009). Hacia una elucidación del campo de estudios sobre cine en Chile. AISTHESIS, N46, 270-283. 